\title{
DESENVOLVIMENTO DE FERRAMENTA DIDÁTICA PARA SIMULAÇÃO DE COLUNA DE DESTILAÇÃO POR MEIO DO MÉTODO DE MCCABE-THIELE
}

\author{
DEVELOPMENT OF DIDACTIC TOOL FOR DISTILLATION COLUMN \\ SIMULATION BY MCCABE-THIELE METHOD
}

\author{
Willbrynner Pereira Marques ${ }^{1 *}$ \\ Ricardo Santório ${ }^{2}$ \\ ${ }^{1}$ Universidade Federal do Espírito Santo (UFES), Campus São Mateus, São Mateus-ES. E-mail: \\ will.pm3@hotmail.com \\ ${ }^{2}$ Faculdade Pitágoras de Linhares, Linhares-ES. E-mail: ricardo.santorio@pitagoras.com.br \\ *Autor para correspondência.
}

Artigo submetido em 30/09/2018, aceito em 27/12/2018 e publicado em 15/08/2019.

Resumo: A destilação é provavelmente um dos processos de separação mais aplicados na indústria química, sendo que a separação das espécies se deve à diferença de volatilidade. Para abordagens práticas no ensino de engenharias, professores têm buscado desenvolver novas metodologias de ensino. Um algoritmo para a determinação do ponto de orvalho de uma mistura binária foi proposto, sendo aplicado a uma análise numérica do método gráfico de McCabe-Thiele para determinação do número de estágios teóricos e o estágio ótimo de alimentação. Os diagramas de equilíbrio líquido-vapor foram desenvolvidos com base na lei de Raoult modificada, junto com a equação de Antoine para a determinação das pressões de vapor das espécies. Já para a predição do coeficiente de atividade, foi utilizada a equação de Wilson. A partir dos algoritmos montados, foi desenvolvido uma ferramenta didática para simulação de colunas de destilação. Os resultados obtidos para o diagrama ELV de misturas binárias pelo algoritmo implementado foram comparados com os do ChemSep, sendo verificado pouco desvio entre os dados analisados. Foi feito um estudo de caso para uma coluna de destilação que separa uma mistura binária a fim de avaliar se o algoritmo implementado para determinação do número de estágios teóricos e o estágio ótimo de alimentação é satisfatório.

Palavras-chave: coluna de destilação; McCabe-Thiele; VBA; ferramenta didática.

Abstract: Distillation is probably one of the most applied separation processes in the chemical industry,
since the separation of species is due to the difference in volatility. Professors have been trying to
develop new teaching methodologies in order to have more practical approaches for Engineering. For
the prediction of the coefficient of activity, the Wilson equation was used. An algorithm for the
determination of the dew point of a binary mixture was proposed and applied to a numerical analysis of
the McCabe-Thiele graphical method to determine the number of theoretical stages and the optimal stage
of feeding. The liquid-vapor equilibrium diagrams were developed based on the modified Raoult's law,
along with the Antoine equation for the determination of the vapor pressures of the species. From the
assembled algorithms, a didactic tool was developed to simulate distillation columns. The results
obtained for the ELV diagram of binary mixtures by the implemented algorithm were compared with
those of the ChemSep, being verified little deviation between the data analyzed. A case study was carried
out for a distillation column separating a binary mixture in order to evaluate if the algorithm 
implemented to determine the number of theoretical stages and the optimal stage of feeding is satisfactory.

Keywords: distillation column; McCabe-Thiele; VBA; didactic tool.

\section{INTRODUÇÃO}

A destilação é provavelmente um dos processos de separação mais aplicados na indústria química, sua aplicação vai desde a retificação do álcool até o refinamento do petróleo (TOWLER; SINNOTT, 2009). Essa operação unitária é utilizada para separar componentes de misturas líquidas pela diferença de volatilidade (GEANKOPLIS, 1993).

A análise, projeto, operação, controle e otimização de colunas de destilação foram estudados extensivamente no último século, mas, até a introdução dos computadores, somente cálculos manuais e métodos gráficos foram desenvolvidos e aplicados no estudo da destilação. Como a análise da destilação envolve muitos cálculos iterativos de equilíbrio de fases líquido-vapor e balanços massas em diversos estágios da coluna de destilação que são realizados de forma mais acurada e rápido através da computação, o uso de computadores tem tido um efeito benéfico nas recentes décadas. (KISS, 2013).

Como a destilação é um processo que demanda grande quantidade de energia, é desejável determinar algumas variáveis de processo a fim de minimizar custos de operação (GANI; BEK-PEDERSEN, 2000). $O$ gasto energético do processo de destilação está relacionado à baixa eficiência termodinâmica do processo que ocorre devido à grande diferença de temperatura entre o refervedor e o condensador (PONCE et al., 2015).

De acordo com GUILLAUME et al. (2017), é possível separar em três partes o processo de projetar uma coluna de destilação. Primeiro deve-se determinar se o projeto é viável do ponto de vista dos balanços de massa e termodinâmico. Posteriormente, define-se alguns parâmetros chaves, como o estágio de alimentação, a razão de refluxo mínimo e o número de estágios teóricos da coluna. Por fim, faz-se uma simulação da coluna com um software.

Uma solução no projeto de colunas de destilação foi proposta por MCCABE e THIELE (1925), os quais desenvolveram um método gráfico rápido e acurado capaz de descrever o número de pratos necessários para uma coluna separar uma certa mistura binária. Os diagramas de McCabe-Thiele podem ser construídos pela interpolação dos dados de fração molar das espécies na fase líquida e vapor. Esses dados podem ser obtidos através de modelos termodinâmicos (MATHIAS, 2009).

Os cálculos pelo método de McCabeThiele podem ser realizados pelo método gráfico, que é realizado à mão e pode demandar tempo, ou por meio de um software comercial, o qual pode necessitar de uma licença e gerar custos (DONGARE et al., 2016).

Para abordagens práticas no ensino de engenharias, professores têm buscado desenvolver novas metodologias de ensino. RASTEIRO e URBANO (2017) avaliaram a reação de alunos ao utilizarem um portal de ensino online em um curso de engenharia química. Foi observado que embora os estudantes não tenham sidos obrigados a utilizar a ferramenta, eles sentiram-se dispostos e interessados a fazê-lo.

O presente trabalho propõe-se a desenvolver algoritmos que realizem uma análise numérica do método gráfico de McCabe-Thiele a fim de determinar o número de pratos teóricos e o estágio ótimo de alimentação de uma coluna de destilação. Os algoritmos foram implementados no VBA (Visual Basic for Applications) e criado uma GUI (Graphics User Interface Interface Gráfica do Usuário) de forma que a 
ferramenta possa ser utilizada como apoio didático.

\section{MATERIAIS E MÉTODOS}

Para a determinação do ponto de orvalho de uma mistura binária foi proposto uma sub-rotina (Figura 1) a qual resolve as equações da lei de Raoult modificada, a equação de Antoine e a equação de Wilson, usando um método iterativo. Esse algoritmo se faz necessário para determinação das composições das fases líquido e vapor em cada prato da coluna de destilação, sendo incorporado ao algoritmo que avalia o número de estágios teóricos e o estágio ótimo de alimentação por uma solução numérica baseada no método gráfico de McCabeThiele (Figura 4).

\subsection{LEI DE RAOULT MODIFICADA}

A fim de desenvolver o diagrama de equilíbrio líquido-vapor para misturas binárias, utiliza-se no algoritmo a lei de Raoult modificada, já que grande parte das misturas apresentam desvios da idealidade. Por exemplo, o comportamento da pressão de vapor de uma mistura etanol-água desvia consideravelmente da Lei de Raoult (BERK, 2013). Portanto, faz-se necessária a inserção do coeficiente de atividade, $\gamma$, na equação de Raoult.

$$
\mathrm{y}_{\mathrm{i}} \mathrm{P}=\mathrm{x}_{\mathrm{i}} \gamma_{\mathrm{i}} \mathrm{P}_{\mathrm{i}}^{\circ}
$$

Sendo $x_{i}$ e $y_{i}$ a fração molar da espécie i na fase líquida e na fase vapor, respectivamente.

\subsubsection{Equação de Antoine}

A equação de Antoine foi utilizada no algoritmo a fim de relacionar a pressão de vapor e a temperatura e é representada por

$$
\log \mathrm{P}_{\mathrm{i}}^{\circ}=\mathrm{A}_{\mathrm{i}}-\frac{\mathrm{B}_{\mathrm{i}}}{\mathrm{T}+\mathrm{C}_{\mathrm{i}}}
$$

onde $\mathrm{P}_{\mathrm{i}}^{\circ}$ é a pressão de vapor da espécie $\mathrm{i}(\mathrm{em}$ $\mathrm{mmHg}), \mathrm{T}$ é a temperatura $\left(\mathrm{em}^{\circ} \mathrm{C}\right)$ e $\mathrm{A}_{i}, \mathrm{~B}_{\mathrm{i}} \mathrm{e}$
$\mathrm{C}_{\mathrm{i}}$ são constantes para a espécie i (GREEN; PERRY, 2007).

\subsubsection{Predição do coeficiente de atividade}

A equação de Wilson é um método de predição de coeficientes de atividade binários bastante utilizado. GHOSH e CHOPRA (1975) destacam que a equação de Wilson tem como plano de fundo a teoria de Flory-Huggin para soluções atérmicas e envolve duas constantes que independem da temperatura para cada par binário, sendo mais conveniente sua utilização (HOLMES; WINKLE, 1970).

Para um sistema binário, a equação de Wilson é dada por

$$
\begin{aligned}
& \ln \gamma_{1} \\
& =-\ln \left(\mathrm{x}_{1}+\Lambda_{12} \mathrm{x}_{2}\right) \\
& +\mathrm{x}_{2}\left(\frac{\Lambda_{12}}{\mathrm{x}_{1}+\Lambda_{12} \mathrm{x}_{2}}\right. \\
& \left.-\frac{\Lambda_{21}}{\mathrm{x}_{2}+\Lambda_{21} \mathrm{x}_{1}}\right) \\
& \ln \gamma_{2} \\
& =-\ln \left(\mathrm{x}_{2}+\Lambda_{21} \mathrm{x}_{1}\right) \\
& -\mathrm{x}_{1}\left(\frac{\Lambda_{12}}{\mathrm{x}_{1}+\Lambda_{12} \mathrm{x}_{2}}\right. \\
& \left.-\frac{\Lambda_{21}}{\mathrm{x}_{2}+\Lambda_{21} \mathrm{x}_{1}}\right)
\end{aligned}
$$

onde $\gamma_{1}$ e $\gamma_{2}$ é o coeficiente de atividade da espécie 1 e 2, respectivamente. Os parâmetros de Wilson $\Lambda_{12}$ e $\Lambda_{21}$ são expressos como

$$
\Lambda_{\mathrm{ij}}=\frac{\mathrm{v}_{\mathrm{j}}^{\mathrm{L}}}{\mathrm{v}_{\mathrm{i}}^{\mathrm{L}}} \exp \left(-\frac{\lambda_{\mathrm{ij}}-\lambda_{\mathrm{ii}}}{\mathrm{RT}}\right)
$$

$v_{\mathrm{i}}^{\mathrm{L}}$ e $v_{\mathrm{j}}^{\mathrm{L}}$ é o volume molar da espécie i e j na fase líquida.

Parâmetros para a equação de Wilson de diversas misturas binárias podem ser 
obtidas no estudo realizado por HOLMES e WINKLE (1970).

\subsubsection{Ponto de orvalho}

SMITH et al. (2005) descrevem o ponto de orvalho como sendo aquele no qual as últimas gotas de líquido desaparecem. A temperatura de condensação e a fração das espécies na fase líquida nesse ponto são obtidas pela solução do sistema de equação que contempla a equação de Raoult modificada, a equação de Antoine e a equação de Wilson por um método iterativo, conforme Figura 1.

Figura 1: Algoritmo utilizado para a determinação do ponto de orvalho à pressão constante

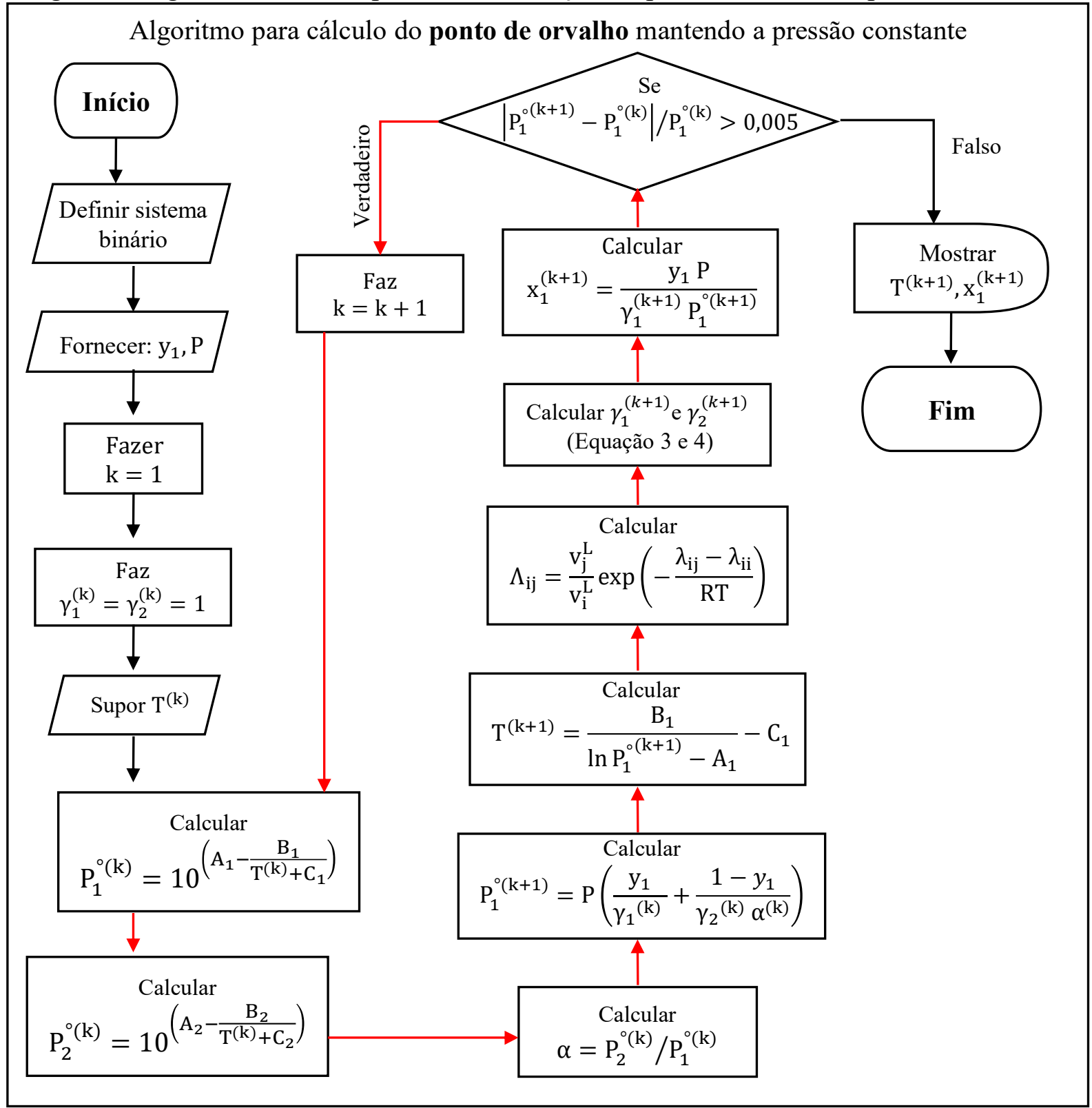

Fonte: Elaborada pelo autor. 


\subsection{BALANÇO DE MATERIAL DA COLUNA}

A partir dos balanços de material realizados para uma coluna de destilação (Figura 2), pode-se obter as equações da linha da seção de esgotamento e retificação conforme equação (6) e (7), respectivamente.

$$
\begin{aligned}
y_{m}=\left(\frac{R_{B}+1}{R_{B}}\right) & x_{m-1} \\
& -\left(\frac{1}{R_{B}}\right) x_{B}
\end{aligned}
$$

$$
\begin{aligned}
y_{n+1}=\left(\frac{R_{D}}{R_{D}+1}\right) x_{n} \\
+\left(\frac{1}{R_{D}+1}\right) x_{D}
\end{aligned}
$$

Nesse modelo considera-se que o condensador é total, ou seja, não conta como um estágio de equilíbrio, enquanto que o refervedor é parcial, contando como um estágio de equilíbrio.

Figura 2: Esquema de uma coluna de destilação fracionada.

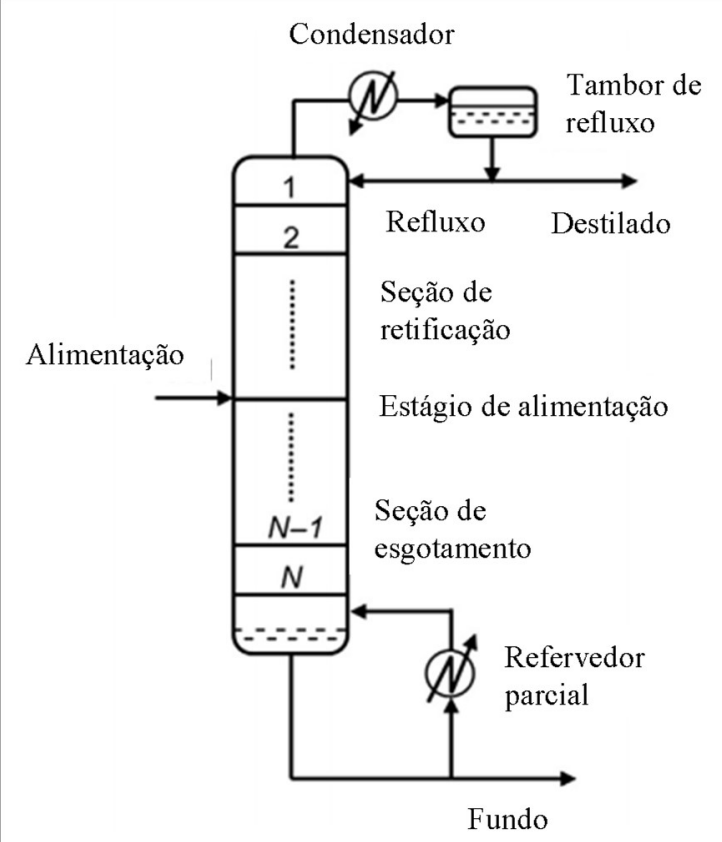

Fonte: Adaptada de GORAK e SORENSEN (2014).

\subsubsection{Considerações para seção de alimentação}

A equação da linha de operação da seção de alimentação é fornecida pela equação (8).

$$
\mathrm{y}=\left(\frac{q}{q-1}\right) \mathrm{x}-\left(\frac{1}{q-1}\right) \mathrm{z}_{\mathrm{F}}
$$

Onde $q$ é uma medida da condição térmica da alimentação, representando a quantidade de mols de líquido saturado formado no estágio de alimentação por mol de alimentação (GREEN; PERRY, 2007). $\mathrm{Na}$

Figura 3 são apresentados os valores atribuídos a $q$ de acordo com a condição na seção de alimentação. 
Figura 3: Efeitos das condições térmicas da linha q.

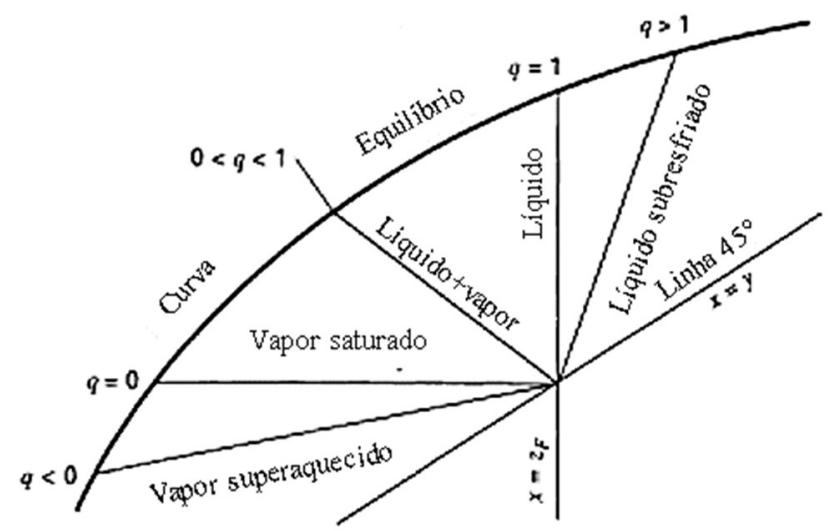

Fonte: Adaptada de SEADER e HENLEY (2006).

\subsubsection{Intersecção entre linhas de operação}

A transição entre a seção de retificação e de esgotamento se dá na interseção entre as linhas de operação. Ao combinar a equação (7) com a (8) pode-se gerar uma nova equação quando $q \neq 0$ e $q \neq$ 1 para determinar a fração da espécie na fase líquida na transição da seção conforme equação (9).

$$
x_{\text {int }}=\frac{x_{D}(q-1)-z_{F}\left(R_{D}+1\right)}{R_{D}(q-1)-q\left(R_{D}+1\right)}
$$

Para obter a fração da espécie na fase vapor na transição da seção a equação (8) pode ser reescrita da seguinte forma

$$
\begin{aligned}
y_{\text {int }}=\left(\frac{R_{D}}{R_{D}+1}\right) & x_{\text {int }} \\
& +\left(\frac{1}{R_{D}+1}\right) x_{D}
\end{aligned}
$$

Para $\mathrm{q}=0$ tem-se que $\mathrm{y}_{\text {int }}=\mathrm{z}_{\mathrm{F}}$, sendo que $\mathrm{x}_{\text {int }}$ pode ser calculado pela equação (7) fazendo um rearranjo matemático.

$$
\begin{aligned}
x_{\text {int }}=\left(1+\frac{1}{R_{D}}\right) y_{\text {int }} \\
-\left(\frac{1}{R_{D}}\right) x_{D}
\end{aligned}
$$

Para $q=1$ tem-se que $x_{\text {int }}=z_{F}$, sendo $y_{\text {int }}$ obtido pela equação (10).

$\mathrm{Na}$ Figura 4 é apresentado o algoritmo que engloba a sub-rotina para determinação da composição das fases em equilíbrio, a determinação das linhas de operação e das composições em cada estágio teórico. 
Figura 4: Algoritmo utilizado para a determinação do número de estágios teóricos e o estágio ótimo de alimentação para uma coluna de destilação

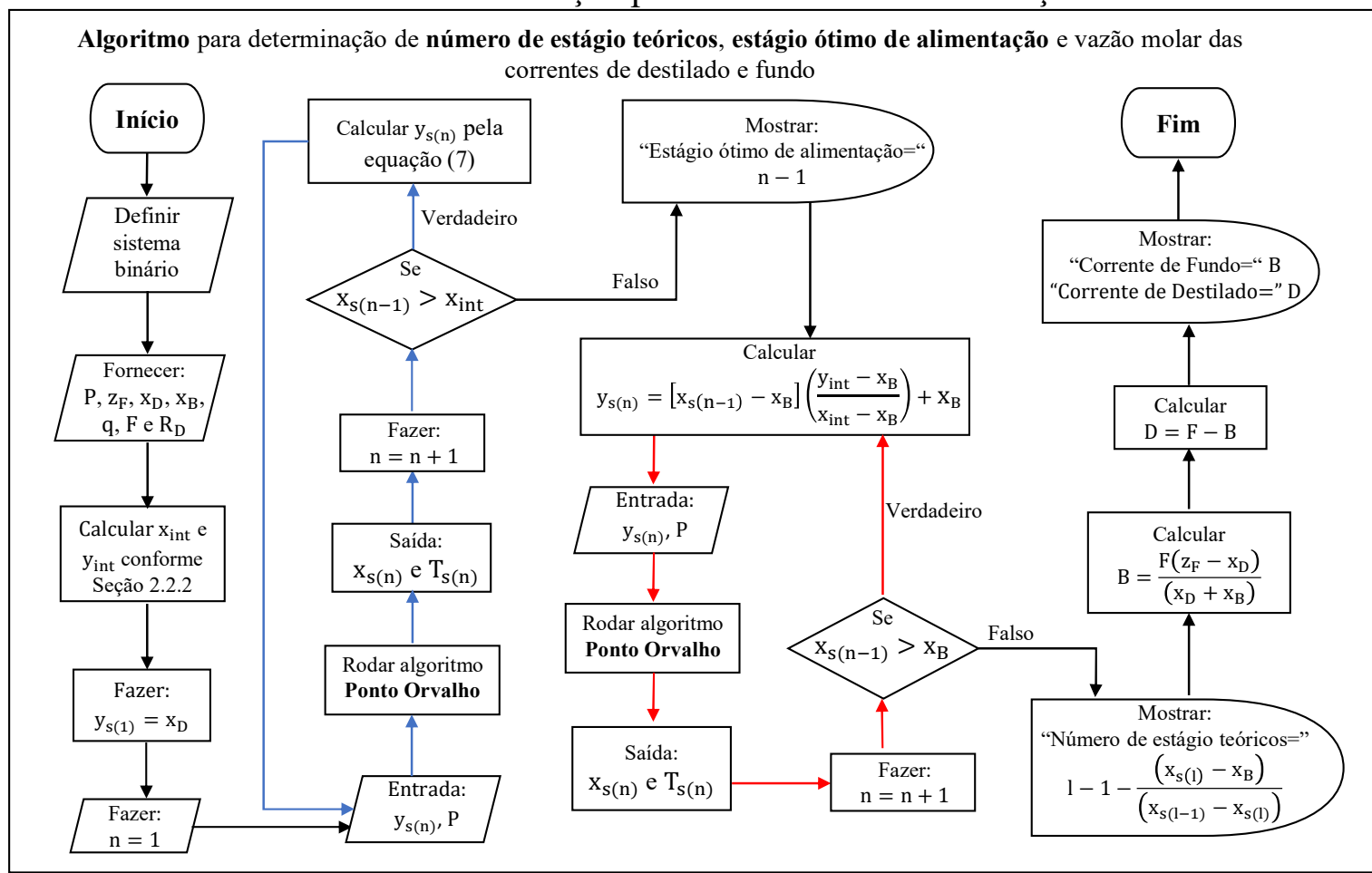

Fonte: Elaborada pelo autor.

\section{RESULTADOS E DISCUSSÃO}

A interface gráfica desenvolvida para a ferramenta educacional pode ser observada na

Figura 5. Para facilitar o uso da ferramenta, a janela foi dividida em duas partes. $\mathrm{Na}$ primeira parte, intitulada como "Dados para Gerar Gráfico ELV", define-se a mistura binária que será separada bem como a pressão em que a coluna de destilação opera. Já na segunda parte, determinada "Método de McCabe-Thiele", insere-se os parâmetros necessários para traçar as linhas de operação e dos estágios teóricos. Ao alterar as variáveis relacionadas com as linhas de operação, a figura com o diagrama de equilíbrio instantaneamente é atualizada de forma que o usuário possa avaliar os efeitos na modificação desses parâmetros.

$\mathrm{Na}$ Figura 6 é possível comparar os resultados obtidos pelo algoritmo implementado no VBA e aqueles fornecidos pelo ChemSep, os quais apresentam baixo desvio entre si. No material suplementar (Figura S1) é mostrado diagramas ELV para outras misturas binárias obtidas pelo algoritmo implementado.

Na Figura 7 é mostrado o resultado da simulação de uma coluna de destilação que separa uma mistura binária constituída por etanol e água, sendo a coluna mantida a uma pressão de $101,325 \mathrm{kPa}$. A Figura S2 do material suplementar mostra a interface do programa com o resultado da simulação. As condições nessa coluna são:

- Fração de etanol na alimentação $=0,4$ [mol/mol]

- Fração de etanol na corrente de fundo $=0,05[\mathrm{~mol} / \mathrm{mol}]$

- Fração de etanol na corrente de destilado $=0,8[\mathrm{~mol} / \mathrm{mol}]$

- Razão de refluxo no destilado, $\mathrm{R}_{\mathrm{d}}=$ 1,7

- Parâmetro $\mathrm{q}=1$ (alimentação é constituída de líquido saturado)

Verificou-se que o número de estágios teóricos são 10,08 , enquanto que o 
estágio ótimo de alimentação é o $9^{\circ}$. No material suplementar são apresentados outros dois casos que foram simulados.

Figura 5: Interface da ferramenta educacional

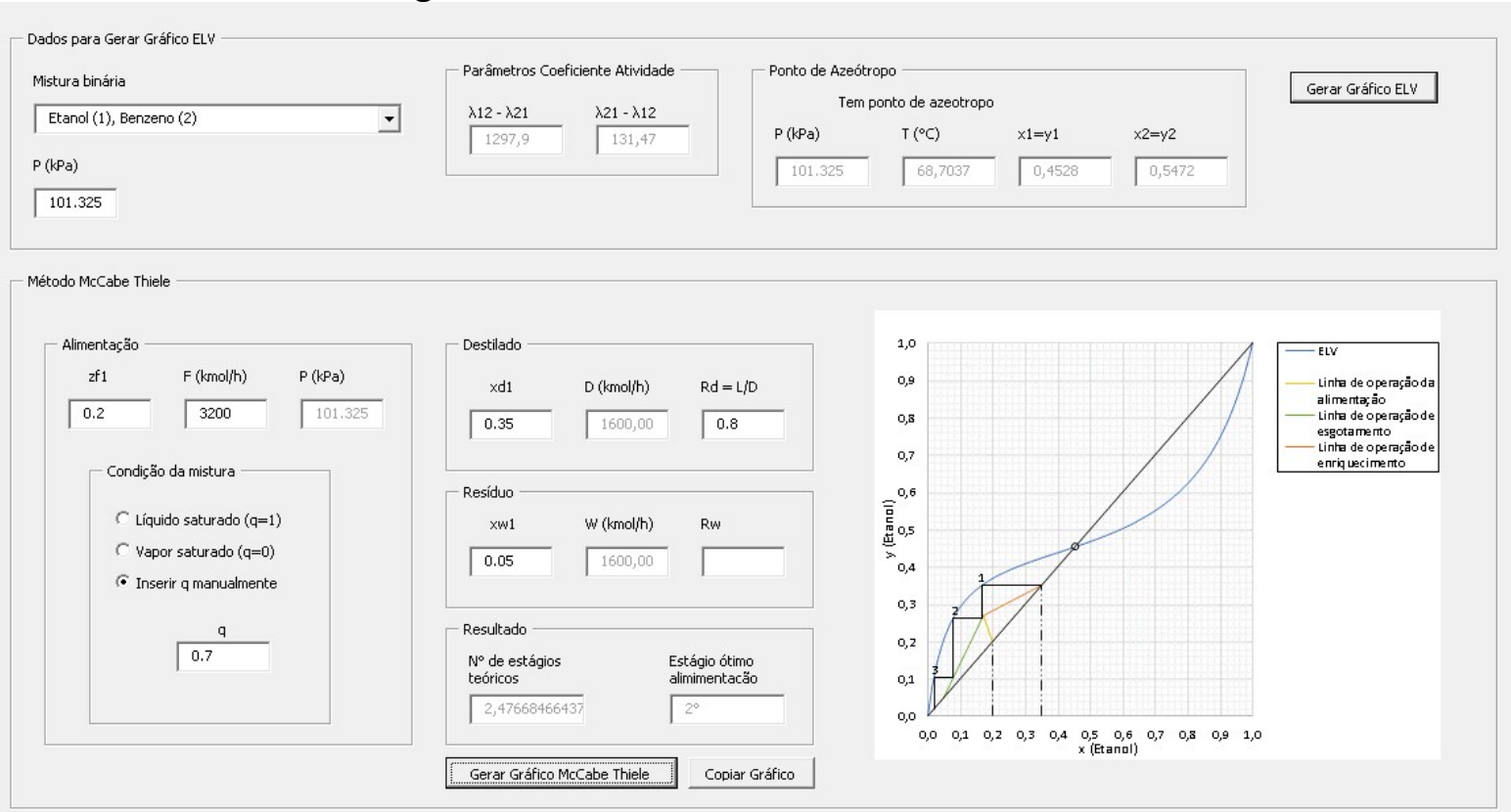

Fonte: Elaborada pelo autor.

Figura 6: Comparação do diagrama de equilíbrio líquido-vapor para uma mistura etanol-água à $760 \mathrm{mmHg}$ obtidos pelo algoritmo implementado e o Chemsep.

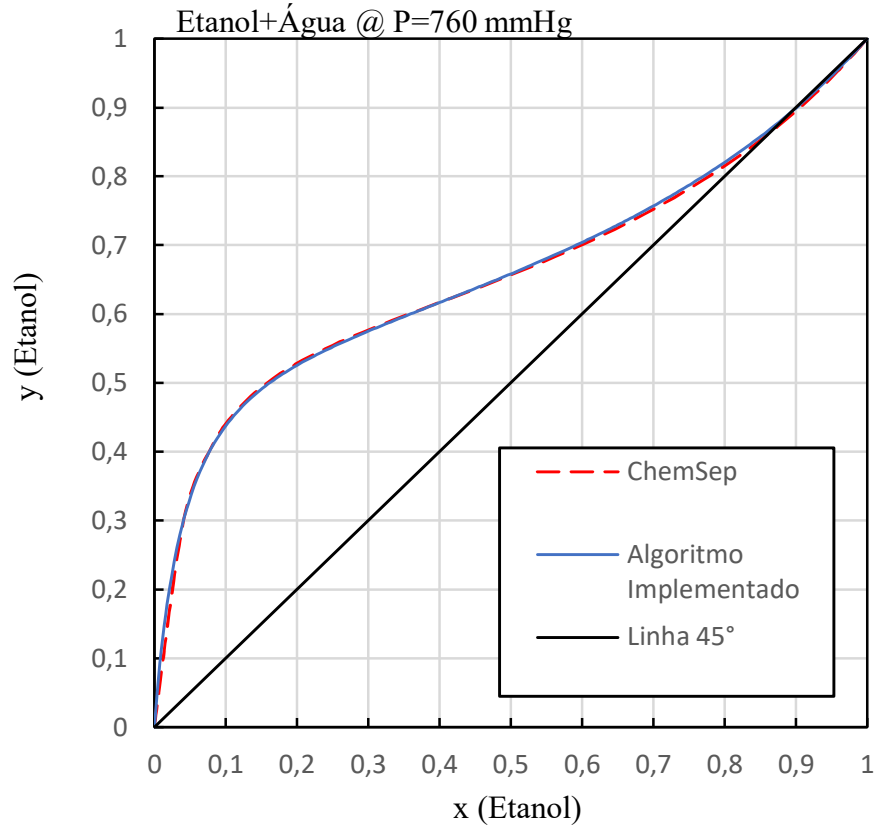

Fonte: Elaborada pelo autor. 
Figura 7: Resultado de simulação para coluna de destilação que processa uma mistura etanolágua em $\mathrm{P}=101,325 \mathrm{kPa}$

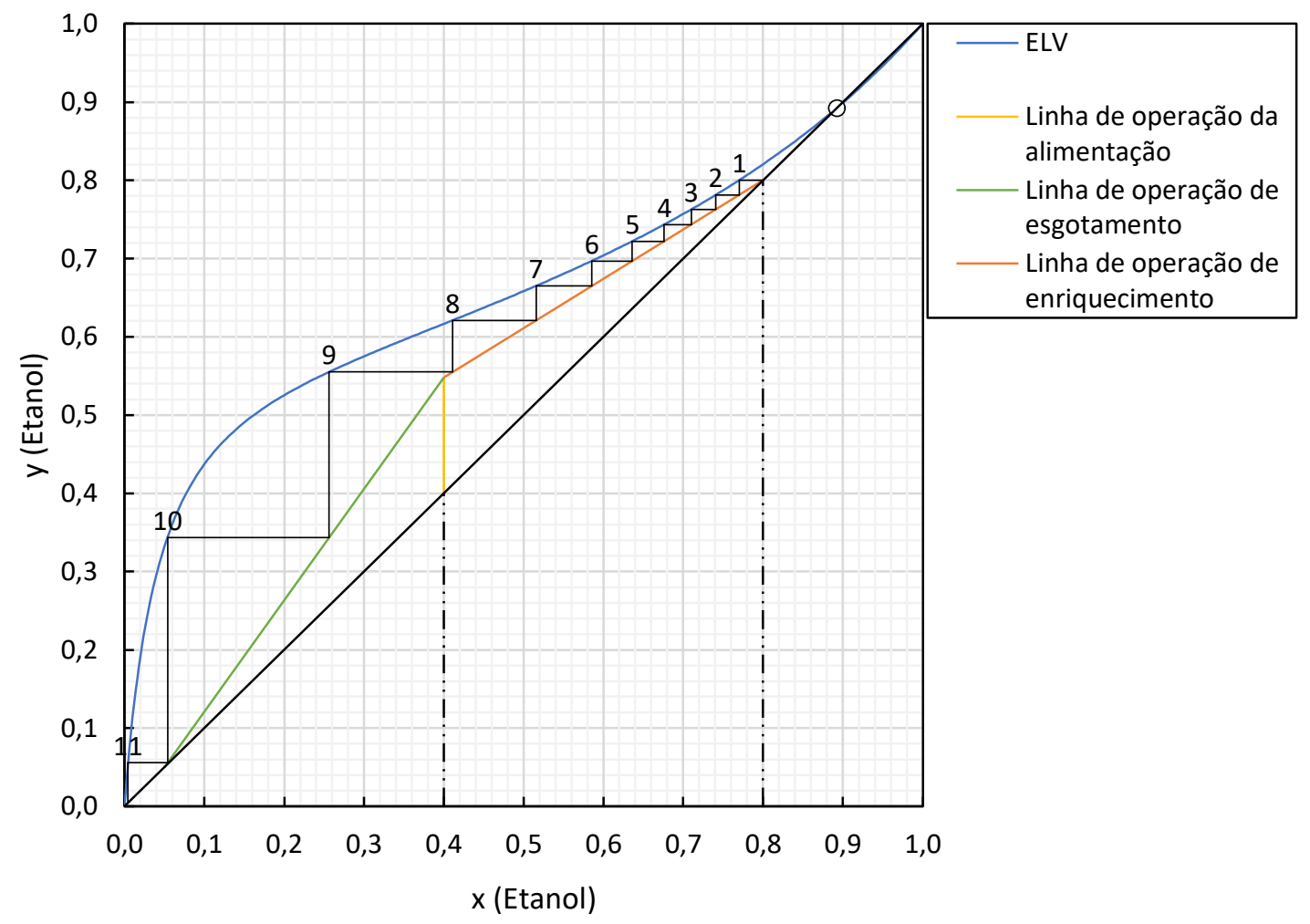

Fonte: Elaborada pelo autor.

\section{CONCLUSÕES}

Nesse trabalho foi desenvolvido um algoritmo para determinação do número de estágios teóricos e o estágio ótimo de alimentação por meio de uma solução numérica baseado no método gráfico de McCabe-Thiele. Esse algoritmo tem como proposta uma apresentação didática dessa importante área da engenharia química, bem como promover uma solução mais precisa para simulação de colunas de destilação que separam misturas binárias.

Para isso, primeiramente construiu-se um algoritmo para obtenção do diagrama de equilíbrio líquido-vapor para uma mistura binária utilizando a equação de Raoult modificada, a equação de Antoine e a equação de Wilson. Os resultados dos diagramas obtidos foram comparados com aqueles coletados no Chemsep, sendo observado um pequeno desvio entre ambos os dados.

A fim de avaliar o algoritmo baseado no método McCabe-Thiele, foram simulados alguns estudos de casos, tendo um resultado satisfatório. Além disso, foi criado uma interface gráfica para uma ferramenta educacional utilizando os algoritmos desenvolvidos.

Sugere-se desenvolver um algoritmo para determinação da razão de refluxo mínimo, visto que a otimização do custo fixo anual e o custo operacional de uma coluna de destilação está relacionada com uma relação entre a razão de refluxo e a razão mínima de refluxo. 


\section{REFERÊNCIAS}

BERK, Z. Food Process Engineering and Technology. 2nd Editio ed. [s.1.] Academic Press, 2013.

DONGARE, S. B. et al. Shortcut Design Method for Multistage Binary Distillation via MS-Excel. Journal of Engineering Research and Application, v. 6, n. 10, 2016.

GANI, R.; BEK-PEDERSEN, E. Simple new algorithm for distillation column design. AIChE Journal, v. 46, n. 6, p. 1271-1274, jun. 2000.

GEANKOPLIS, C. J. Transport Processes and Unit Operations. [s.1.] Prentice Hall, 1993.

GHOSH, S. K.; CHOPRA, S. J. Activity Coefficients from the Wilson Equation. Industrial \& Engineering Chemistry Process Design and Development, v. 14, n. 3, p. 304-308, jul. 1975.

GORAK, A.; SORENSEN, E. Distillation: Fundamentals and Principles. 1st Editio ed. [s.1.] Academic Press, 2014.

GREEN, D. W.; PERRY, R. H. Perry's Chemical Engineers' Handbook. 8. ed. [s.1.] McGraw-Hill Professional Publishing, 2007.

GUILLAUME, W. et al. The production zone method: A non-ideal shortcut method for the design of distillation columns.

Separation and Purification Technology, v. 184, p. 404-423, ago. 2017.

HOLMES, M. J.; WINKLE, M. V. PREDICTION OF TERNARY VAPORLIQUID EQUILIBRIA FROM BINARY DATA. Industrial \& Engineering Chemistry, v. 62, n. 1, p. 21-31, jan. 1970.

KISS, A. A. Advanced distillation technologies: design, control, and applications. [s.1.] Wiley, 2013.

MATHIAS, P. M. Visualizing the McCabeThiele Diagram. Chemical Engineering Progress, v. 105, 2009.

MCCABE, W. L.; THIELE, E. W.
Graphical Design of Fractionating Columns. Industrial \& Engineering Chemistry, v. 17, n. 6, p. 605-611, jun. 1925.

PONCE, G. H. S. F. et al. Using an internally heat-integrated distillation column for ethanol-water separation for fuel applications. Chemical Engineering Research and Design, v. 95, p. 55-63, mar. 2015.

RASTEIRO, M. G.; URBANO, D.

Evaluation of on-line simulation tools to teach Chemical Processes. 2017 4th Experiment@International Conference (exp.at'17). Anais...IEEE, jun. 2017Disponível em: http://ieeexplore.ieee.org/document/798439 7I

SEADER, J. D.; HENLEY, E. J. Separation Process Principles. 2nd Editio ed. [s.1.] John Wiley \& Sons, 2006.

SMITH, J. M.; NESS, H. C. VAN; ABBOTT, M. M. Introduction to Chemical Engineering Thermodynamics. 7th editio ed. [s.1.] McGraw Hill Higher Education, 2005.

TOWLER, G.; SINNOTT, R. Chemical Engineering Design. 5th Editio ed. [s.1.] Butterworth-Heinemann, 2009. 